\title{
TIPE2 governs macrophage polarization via negative regulation of $\mathrm{mTORC1}$
}

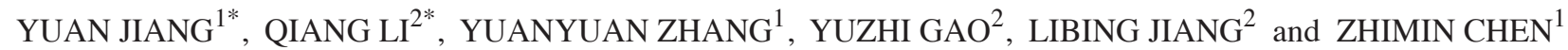 \\ ${ }^{1}$ Department of Pulmonology, Children's Hospital, Zhejiang University School of Medicine, Hangzhou, Zhejiang 310051; \\ ${ }^{2}$ Emergency Department, Second Affiliated Hospital of Zhejiang University School of Medicine, \\ Hangzhou, Zhejiang 310009, P.R. China
}

Received February 17, 2017; Accepted October 6, 2017

DOI: $10.3892 / \mathrm{mmr} .2017 .7991$

\begin{abstract}
Macrophages can be polarized into the inflammatory M1 lineage or the immunomodulatory M2 lineage, depending on the differential tissue microenvironment signaling, specific pathogens or cytokine stimulation. Tumor necrosis factor $\alpha$-induced protein 8 -like protein 2 (TIPE2) has been demonstrated to negatively regulate inflammation by inhibiting the Toll-like receptor (TLR) pathway. The present study utilized murine bone marrow derived macrophages (BMDMs) as the model of undifferentiated (M0) macrophages to study the roles of TIPE2 in the differential polarization status of BMDMs. It was observed that the expression levels of TIPE2 were diminished in M1 macrophages treated with lipopolysaccharide/interferon $\gamma$, and elevated in M2 macrophages treated with interleukin (IL)-4. BMDMs with TIPE2 overexpression exhibited defective M1 polarization and enhanced responses to IL-4 stimulation. TIPE2 impeded M1 polarization by interfering with mitogen-activated protein kinase kinase kinase 7-inhibitor of nuclear factor- $\kappa \mathrm{B}$ kinase subunit $\beta$ and B cell receptor-associated protein-serine/threonine-protein kinase mTOR complex 1 (mTORC1) activation. TIPE2 overexpression accelerated IL-4 induced M2 polarization by dampening mTORC1 activation via the accelerated process of arginine to urea. Overall, these results define a key role for TIPE2 in macrophage polarization by impeding mTORC1 response.
\end{abstract}

Correspondence to: Dr Zhimin Chen, Department of Pulmonology, Children's Hospital, Zhejiang University School of Medicine, 3333 Binsheng Road, Hangzhou, Zhejiang 310051, P.R. China

E-mail: drchenzm@163.com

${ }^{*}$ Contributed equally

Key words: tumor necrosis factor $\alpha$-induced protein 8-like protein 2, macrophage polarization, serine/threonine-protein kinase mTOR complex 1 , arginine

\section{Introduction}

Macrophages serve different roles in homeostasis, innate immunity against microbes and tissue repair (1-3). Macrophages are differentiated from monocytes, and maybe polarized into the inflammatory M1 (classically activated) lineage or the immunomodulatory M2 (alternatively activated) lineage when they leave the blood vessels, depending on differential tissue microenvironment signaling, specific pathogens or cytokine stimulation $(2,4)$. M1 polarization is induced by lipopolysaccharide (LPS) and/or interferon- $\gamma$ (IFN- $\gamma$ ) during acute infections to release pro-inflammatory cytokines and chemokines that mount an immune response against various intracellular pathogens, whereas M2 polarization is primarilyactivated by interleukin-4 (IL-4) to produce anti-inflammatory cytokines (e.g., IL-10), provide tissue remodeling and enhance adaptive $\mathrm{T}$ helper 2 cell immunity $(4,5)$.

Previous studies intomacrophage function suggested that the activation of serine/threonine-protein kinase mTOR complex 1 (mTORC1) and mTORC2 may selectively control differential macrophage polarization (6-8). Macrophages with constitutive mTORC1 activation were resistant to M2 polarization, although they displayed elevated M1 inflammation via mTORC1-mediated downregulation of RAC- $\alpha$ serine/threonine-protein kinase (Akt) signaling under IL-4 stimulation (6). Conversely, the mTORC2-interferon regulatory factor (IRF) 4 pathway was important for IL-4 induced alternative M2 activation (9). Additionally, mTORC2-Akt suppressed LPS-Toll like receptor 4 (TLR4) induced inflammation in innate immunity (10). Paradoxically, IL-4 may cooperate with the Akt-mTORC1 pathway to regulate M2 activation via increased M2 gene-specific histone acetylation (11). Whether mTORC1 and mTORC2 are integrally linked during the polarization of macrophages remains unknown. In addition, macrophage polarization may be controlled by other regulatory factors.

Tumor necrosis factor $\alpha$-induced protein 8 -like protein 2 (TIPE2) is a member of the tumor necrosis factor- $\alpha$-induced protein 8 family which performs diverse functions, including the negative regulation of innate and adaptive immunity, transcription factor AP-1 and nuclear factor (NF)- $\mathrm{kB}$ activation and tumor suppression $(12,13)$. Consequently, TIPE2-deficient mice have been demonstrated to exhibit increased M1 inflammation and resistance to M2 polarization, and the 
deficiency may retard systemic lupus erythematosus autoimmunity via macrophage polarization $(14,15)$. Additionally, a cell-permeable TIPE2 protein exerted inhibitory effects on mitogen-activated protein kinase kinase kinase 7 (TAK1) phosphorylation (16). The present study assessed the role of TIPE2 in determining commitment to the process of macrophage activation and polarization. M2 polarization was hallmarked by increased TIPE2 expression during M2-like differentiation. Exogenous overexpression of TIPE2 in M2 macrophages drove global expression of M2-specific cytokines via inhibition of mTORC1. Conversely, the induction of an M1-specific phenotype was impaired in M1 macrophages with ectopic TIPE2 expression by disturbing TAK1-inhibitor of NF- $\mathrm{KB}$ (IKK) and TLR4-mTORC1 activation. The results of the present study provided a basis for understanding how TIPE2 may control macrophage activation and polarization.

\section{Materials and methods}

Bone marrow-derived macrophages (BMDMs). All animal procedures were pre-approved by the Institutional Animal Care and Use Committee of Zhejiang University (Hangzhou, China). The present study used 6-week-old male C57BL/6J mice (supplied by Beijing Vital River Laboratory, Beijing, China; $\mathrm{n}=12 ; 18-25 \mathrm{~g}$ ). Mice were housed at room temperature with ad libitum access to food and water, relative humidity 50-60\% and 12-h day/night cycles. The femurs were removed from the mice following euthanasia with sodium pentobarbital (250 mg/kg; Sigma-Aldrich, Merck KGaA, Darmstadt, Germany), and BM cells were released by gently crushing the bones with a sterile mortar and pestle. For macrophage differentiation, BM-derived cells were plated in petri dishes with RPMI 1640 medium (10\% fetal calf serum; penicillin/streptomycin; 2 mM L-glutamine; Thermo Fisher Scientific, Inc., Waltham, MA, USA) supplemented with macrophage colony-stimulating factor (M-CSF; $20 \mathrm{ng} / \mathrm{ml}$; Sigma-Aldrich; Merck KGaA) containing L929 cell supernatant for 7 days (Fig. 1A). M-CSF differentiated macrophages were harvested and plated in tissue culture dishes for subsequent experiments. For M1-like activation, 0.5-0.7 $\times 10^{6}$ BMDMs were plated in 12-well plates and treated with $10 \mathrm{ng} / \mathrm{ml}$ LPS (Sigma-Aldrich; Merck KGaA) and 100 U/ml IFN- $\gamma$ (Sigma-Aldrich; Merck $\mathrm{KGaA}$ ) and incubated overnight. For M2 polarization, the cells were treated with $10 \mathrm{ng} / \mathrm{ml}$ IL-4 (Sigma-Aldrich; Merck $\mathrm{KGaA}$ ) and incubated overnight.

Western blotting. Cells were washed twice with cold PBS and lysed in $1 \%$ nonidet P-40 buffer with EDTA-free protease inhibitor tablets (Roche Diagnostics, Indianapolis, IN, USA) and aphosphatase inhibitor cocktail (Sigma-Aldrich; Merck $\mathrm{KGaA}$ ). The protein concentration in the cell lysates was determined using a Bradford protein concentration assay kit (Beyotime Institute of Biotechnology, Haimen, China). Equal amounts of protein $(10 \mu \mathrm{g})$ were loaded onto $10 \%$ SDS-PAGE gels (Sigma-Aldrich; Merck KGaA) and subsequently transferred to polyvinylidene fluoride membranes (Thermo Fisher Scientific, Inc.) for immunoblotting with the indicated primary antibodies following $1 \mathrm{~h}$ of blocking with $5 \%$ bovine serum albumin (cat. no. KGY00810; Nanjing KeyGen Biotech Co., Ltd., Nanjing, China) at room temperature, the proteins transferred onto polyvinylidene fluoride membrane were incubated overnight with primary antibody solution against the target protein at $4^{\circ} \mathrm{C}$ and then in the IRDye ${ }^{\circledR} 680 \mathrm{RD}$ or IRDye ${ }^{\circledR} 800 \mathrm{CW}$ conjugated secondary antibody solution for $1 \mathrm{~h}$ in a dark at room temperature. Immunoprecipitation was performed by using Pierce ${ }^{\mathrm{TM}}$ Co-Immunoprecipitation kit (cat. no. 26149; Thermo Fisher Scientific, Inc.). Images of protein bands were visualized using Odyssey ${ }^{\circledR}$ Fc imaging system (LI-COR Biosciences, Lincoln, NE, USA) and analyzed using Odyssey software (version 1.2; LI-COR Biosciences).

Antibodies and reagents. Primary antibodies were purchased from Abcam (Cambridge, UK), including: TIPE2 (cat. no. ab110389), IKK $\beta$ (cat. no. ab124957), phospho-Y188-IKK $\beta$ (cat. no. ab194519), IRF5 (cat. no. ab181553), p65 (cat. no. ab16502), phosphor (p)-S536-p65 (cat. no. ab86299), S6K1 (cat. no. ab32529), p-T389-S6K1 (cat. no. ab2571), TSC1 (cat. no. ab217328), TLR4 (cat. no. ab30667), BCAP (cat. no. ab155077), STAT6 (cat. no. ab32520), p-Y641-STAT6 (cat. no. ab28829), AKT (cat. no. ab8805), p-T308-AKT (cat. no. ab38449), p-S473-AKT (cat. no. ab81283), 4E-BP1 (cat. no. ab2606), phospho-T70-4E-BP1 (cat. no. ab75831), IRS1 (cat. no. ab52167), IRS2 (cat. no. ab134101); all 1:1,000 dilution in 5\% bovine serum albumin (cat. no. KGY00810; Nanjing KeyGen Biotech Co., Ltd.), except for $\alpha$-tubulin (Sigma-Aldrich; Merck $\mathrm{KGaA} ; 1: 5,000)$. Immunoglobulin G-conjugated secondary antibodies were purchased from LI-COR Biosciences (cat. nos. 925-32210 and 925-68070; all 1:10,000 dilution in 5\% bovine serum albumin. For flow cytometry, CD11b-phycoerythrin (BD Biosciences, Franklin Lakes, NJ, USA) and F4/80-fluorescein isothiocyanate (BioLegend, Inc., San Diego, CA, USA) antibodies were used. Rapamycin used at a dose of $100 \mathrm{nM}$ for an overnight incubation was purchased from Sigma-Aldrich (Merck KGaA). Sodium nitroprusside (cat. no. S4059, $0.5 \mathrm{mM}$, overnight), as the chemically distinct nitric oxide (NO) donor, was purchased from Selleck Chemicals (Houston, TX, USA). The target sequences for the TIPE2 gene short hairpin (sh)RNA (5'-CCG TGTAAACCACTCAACTCATCTT-3') and hamartin (TSC1) gene shRNA (5'-TACGTCCAAGGTCGGGCAGGAAGA-3') were designed based on the sequence of mouse TIPE2 cDNA (accession no. NM_027206) and the sequence of mouse TSC1 cDNA (accession no. NM_001289575). The negative control shRNA $(20 \mu \mathrm{M})$, TIPE2 $(20 \mu \mathrm{M})$ and TSC1 shRNAs $(20 \mu \mathrm{M})$ were sequence-confirmed and cloned into the pLL3.7-green fluorescent protein lentiviral vector (Clontech, Mountain View, CA USA). Rescue experiments were performed using the pLV-yellow fluorescent protein lentiviral vector (Addgene, Inc., Cambridge, MA USA) expressing the wild-type TIPE2 gene and the K171T-IKK subunit $\beta$ (IKK $\beta$ ) gene mutation. Lentivirus-based TIPE2 shRNA expression plasmid, wild-type TIPE2 expression plasmid and IKK $\beta$ mutant expression plasmid construction, lentivirus harvesting, and lentivirus titer testing were made purchased from IBSBIO Company (Shanghai, China). The negative control lentiviral particles were also provided by IBSBIO Company. ViraDuctin ${ }^{\mathrm{TM}}$ Lentivirus Transduction kit (LTV-201; Cell Biolabs, San Diego, CA, USA) was used to enhance the transduction of BMDMs with lentiviral particles.

Arginase assay. BMDMs were lysed in 0.1\% Triton X-100 lysis buffer (Sigma-Aldrich; Merck KGaA) with EDTA-free protease 

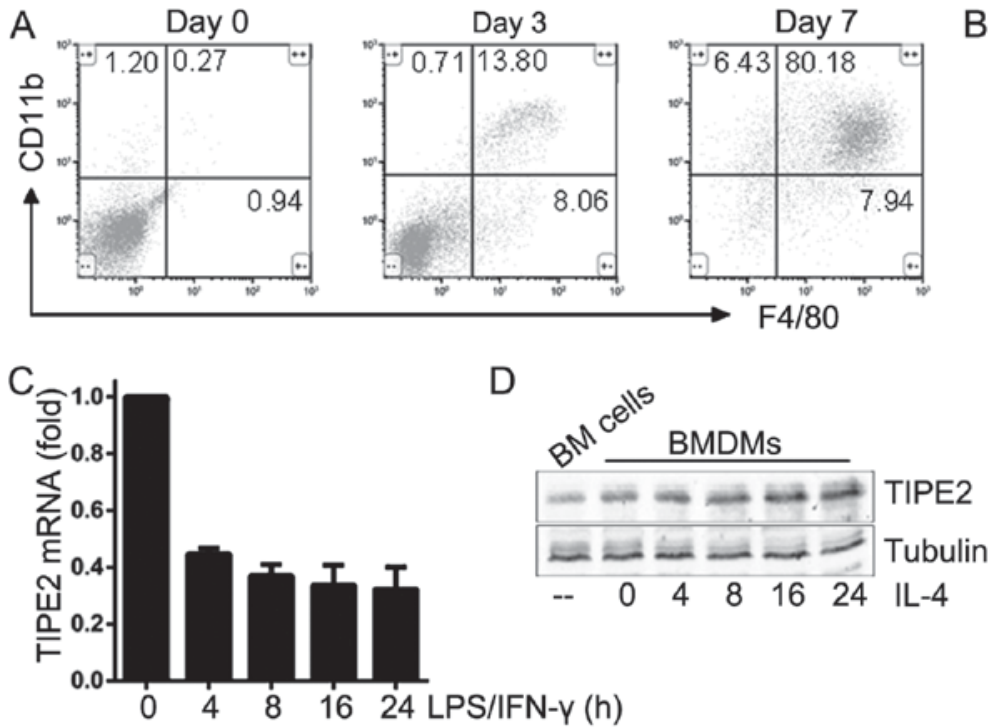

B
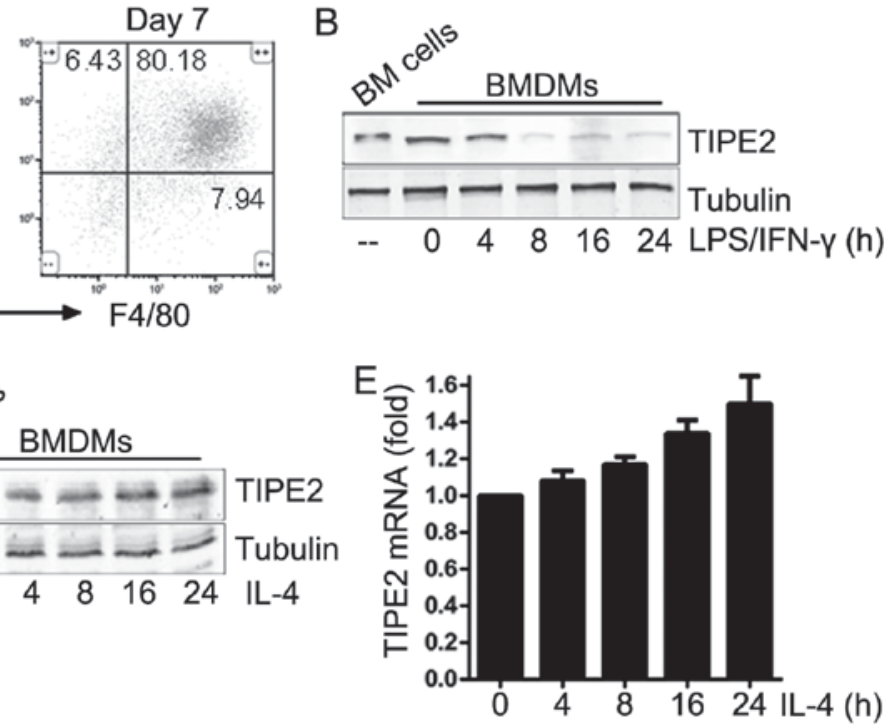

Figure 1. Expression pattern of TIPE2 in murine M1 and M2 macrophages. (A) Flow cytometric analysis of the CD11b ${ }^{+}$, F4/80 ${ }^{+}$macrophage population in $\mathrm{BM}$ cells at days 0,3 and 7 in the presence of macrophage colony stimulating factor $(20 \mathrm{ng} / \mathrm{ml})$. WTBMDMs were stimulated with LPS (10 ng/ml) and IFN- $\gamma$ (100 U/ml) for $24 \mathrm{~h}$. TIPE2 expression levels were determined by (B) immunoblotting and (C) RT-qPCR analysis. WTBMDMs were stimulated with IL-4 $(20 \mathrm{ng} / \mathrm{ml})$ for $24 \mathrm{~h}$. TIPE2 expression levels were determined by (D) immunoblotting and (E) RT-qPCR. Statistics were performed on pooled data from three independent experiments. Error bars represent the standard deviations of the means. TIPE2, tumor necrosis factor $\alpha$-induced protein 8-like protein 2; CD, cluster of differentiation; BMDMs, bone marrow-derived macrophages; LPS, lipopolysaccharide; IFN- $\gamma$, interferon- $\gamma$; RT-qPCR, reverse transcription-quantitative polymerase chain reaction; WT, wild-type; IL-4, interleukin-4.

inhibitor tablets (Roche Diagnostics). Lysates containing equal amounts of protein $(3 \mu \mathrm{g})$ were incubated with $500 \mathrm{mML}$-arginine for $45 \mathrm{~min}$ at $37^{\circ} \mathrm{C}$, followed by acid stop solution. The degradation of L-arginine to urea was measured by adding $9 \%$ isonitrosopropiophenone in $100 \%$ ethanol. Absorbance was read at $540 \mathrm{~nm}$ usinga microplate reader (BioTek Instruments, Inc., Winooski, VT, USA).

ELISA analysis. Cytokine concentration was determined for IL-6 (cat. no. BMS603-2), IL-10 (cat. no. BMS614-2TWO), IL-12b (cat. no. EMIL12B) and tumor necrosis factor- $\alpha$ (TNF $\alpha$, cat. no. BMS607-3) using ELISA kits purchased from Thermo Fisher Scientific, Inc. Experimental supernatants were collected and centrifuged at $4^{\circ} \mathrm{C}$ at $3,000 \mathrm{x}$ g for $5 \mathrm{~min}$. Supernatants were analyzed according to the manufacturer's protocol.

Reverse transcription-quantitative polymerase chain reaction $(R T-q P C R)$. Total RNA was extracted from BMDMs using an RNeasy kit (Qiagen, Inc., Valencia, CA, USA). For RT-qPCR analysis, total RNA (2 $\mu \mathrm{g})$ was converted to cDNA using the SuperScript First-Strand Synthesis system (Takara Bio, Inc., Otsu, Japan). The following thermocycling conditions were used for qPCR: Initial denaturation at $95^{\circ} \mathrm{C}$ for $5 \mathrm{~min}$; followed by 22 cycles of, $95^{\circ} \mathrm{C}$ for $20 \mathrm{sec}, 55^{\circ} \mathrm{C}$ for $20 \mathrm{sec}$ and $72^{\circ} \mathrm{C}$ for $20 \mathrm{sec}$; and final extension for $1 \mathrm{~min}$. The reactions were performed in triplicate with appropriate primers using SYBR-Green (Roche Diagnostics) and an ABI Prism 7900 Sequence Detector (Applied Biosystems; Thermo Fisher Scientific, Inc.). $\beta$-actin was used as the loading control and data were analyzed by the $2^{-\Delta \Delta C q}$ method (17). Sequences for all RT-qPCR primers are presented in Table I.

Statistical analysis. Statistical analysis was performed using Prism 6 (GraphPad Software, Inc., La Jolla, CA, USA) software. The Student's t-test and repeated measures analysis of variance followed by Tukey's post hoc test for multiple comparisons were used to determine statistical significance. Data are presented as the mean \pm standard deviation, $n=3$. $\mathrm{P}<0.05$ was considered to indicate a statistically significant difference.

\section{Results}

Opposite expression patterns of TIPE2 in murine M1 and M2 macrophages. To assess the expression pattern of TIPE2 in the differential polarization of macrophages, BM cells were isolated from C57BL/6J mice. Murine BM cells were exposed to $\mathrm{M}-\mathrm{CSF}$ under appropriate culture conditions to induce differentiation into mature BMDMs. These murine BMDMs were used as the models of undifferentiated (M0) macrophages. RT-qPCR analysis and immunoblotting demonstrated that TIPE2 mRNA and protein expression was impaired $24 \mathrm{~h}$ following LPS/IFN $\gamma$ stimulation of M0 macrophages to induce polarization into M1 macrophages (Fig. 1B and C), whereas IL-4-challenged M0 macrophages polarized into M2 macrophages exhibited elevated TIPE2 expression levels (Fig. 1D and E). Therefore, it was hypothesized that the expression of TIPE2 was associated with M1 and M2 polarization of macrophages.

TIPE2 regulates the differential polarization status of $B M D M s$. As presented in Fig. 1, TIPE2 expression was selectively inhibited during the M1 polarization of macrophages by LPS/IFN $\gamma$, although it was elevated in M2 macrophages. Subsequently, the role of TIPE2 in the process of differential polarization of BMDMs was investigated, and it was observed that BMDMs with TIPE2 shRNA exhibited decreased expression levels of TIPE2 (Fig. 2A). TIPE2 
Table I. Primer sequences used for reverse transcription-quantitative polymerase chain reaction analysis.

\begin{tabular}{lll}
\hline Gene name & Forward primer sequence $\left(5^{\prime} \rightarrow 3^{\prime}\right)$ & Reverse primer sequence $\left(5^{\prime} \rightarrow 3^{\prime}\right)$ \\
\hline$\beta$-actin & TGCGTGACATCAAAGAGAAG & TCCATACCCAAGAAGGAAGG \\
ARG1 & AAGAAAAGGCCGATTCACCT & CACCTCCTCTGCTGTCTTCC \\
ARG2 & GGATCCAGAAGGTGATGGAA & AGAGCTGACAGCAACCCTGT \\
ED38 & ACTGGAGAGCCTACCACGAA 2 & AGTGGGGCGTAGTCTTCTCT \\
FIZZ1 & GATCACAGGCAGGAGAGACTG & GCGAAGCTACTCGGATACGG \\
FPR2 & GGAACTTCTTGCCAATCCAGC & CAGTGGTCCAGTCAACGAGT \\
GPR18 & TTCATGGGCCAGGACTTTCG & CACAGACTTCATGGGGCCTT \\
MGL1 & CTTCGTGCCCTTCCACATCT & TGCGAACACTGCGAAGGTAA \\
MGL2 & AGCCTGGTAAAGCTCCTTCC & GGGATCCAATCACGGAGACG \\
MRC1 & GGAGTCTCCAAAGTTTGCTCT $1 \beta$ & GGTGCCTAGGTCCCTCCTTA \\
YM1 & GTGGAGTGATGGAACCCCAG & CTGTCCGCCCAGTATCCATC \\
TIPE2 & CTTCCGTTGGCCCAGATAC & CTGCTGGGCCTCTTTCAGTA \\
\end{tabular}

ARG, arginase; CD38, cluster of differentiation 38; EGR2, E3 SUMO protein ligase EGR2; FIZZ1, resistin-like $\alpha$; FPR2, formyl peptide receptor 2; GPR18, N-archidonyl glycine receptor; MGL1, C-type lectin domain family 10 member A; MGL2, monoglyceride lipase; MRC1, macrophage mannose receptor 1 ; PGC1 $\beta$, peroxisome proliferator-activated receptor gamma coactivator 1- $\beta$; YM1, chitinase-like protein 3; TIPE2, tumor necrosis factor $\alpha$-induced protein 8 -like protein 2.

deletion negatively regulated the expression levels of IL-10 and M2 genes in BMDMs stimulated by IL-4 (Fig. 2B and C). M1 cytokines (TNFa, IL-6 and IL-12b) and M1 genes were significantly elevated in BMDMs treated with TIPE2 shRNA and LPS/IFN $\gamma$ (Fig. 2D and E). Exogenous TIPE2 overexpression in BMDMs impeded LPS/IFN $\gamma$-induced M1 inflammation (Fig. 3A-C), whereas these cells exhibited enhanced responses to IL-4 stimulation (Fig. 3D and E).

TIPE2 impedes M1 polarization by interfering with TAK1-IKK $\beta$ and BCAP-mTORC1 activation. The TAK1-IKK $\beta$ pathway promotes M1 macrophage polarization via IRF5 and NF- $\kappa \mathrm{B}$ activation (18-20). It was observed that exogenous TIPE2 was able toinhibit IKK $\beta$, and downstream IRF5 and NF- $\kappa$ B activation (Fig. 4A), which was responsible for the negative regulation of TIPE2 in M1 polarization. M0 macrophages with mutant (mt)-IKK $\beta$ (K171T) were additionally constructed (Fig. 4B), and these cells exhibited M1-like functional activities either prior to or during LPS/IFN $\gamma$ stimulation (Fig. 4C and D). Ectopic TIPE2 expression partially antagonized the LPS/IFN $\gamma$ induced expression of M1 cell markers and cytokines during exogenous co-expression of mt-IKK $\beta$ and TIPE2 in macrophages (Fig. 4C and D), suggesting the presence of alternative pathways for M1 polarization interrupted by TIPE2.

In addition to the canonical IRF5 and NF- $\mathrm{B}$ pathways, TLR4-BCAP-mTORC1 signaling is involved in the M1 polarization of macrophages with LPS/IFN $\gamma$ stimulation (7). It was observed that exogenous TIPE2 disturbed the formation of the TLR4-BCAP complex (Fig. 4E) and subsequently inhibited mTORC1 activation, signifiedby diminished S6K phosphorylation (Fig. 4A), in macrophages following LPS/IFN $\gamma$ stimulation. Following TSC1 deletion in BMDMs (Fig. 4B) with mt-IKK $\beta$, the cells displayed hyperactivated mTORC1, IRF5 and NF-кB (Fig. 4B), and were resistant to the effects of disturbance of ectopic TIPE2 on M1 polarization following LPS/IFN $\gamma$ stimulation (Fig. 4C and D). These data suggested that mTORC1 and IKK $\beta$ activation coordinated M1 polarization.

TIPE2 enhances M2 polarization. The above data indicated that TIPE2 attenuated M1 polarization stimulated by LPS/IFN $\gamma$ via the inhibition of IKK $\beta$ and mTORC1 activation. IL-4 induced M2 polarization was subsequently examined, which relied on tyrosine-protein kinase JAK-signal transducer and activator of transcription (STAT)6, and insulin receptor substrate (IRS)1/2-mTORC2-Akt signaling. Constitutive mTORC1 activation impairs M2 polarization by IL-4 (6). It was observed that the activation of STAT6 was unaltered in wild-type (WT) and TIPE2-overexpressing BMDMs (Fig. 5A). However, mTORC2 induced AKT S473 phosphorylation. AKT T308 phosphorylation in BMDMs with TIPE2 overexpression was more marked compared with WT BMDMs (Fig. 5A). IL-4 induced BMDMs with TIPE2 overexpression additionally exhibited weaker phosphorylation of the mTORC1 downstream molecules ribosomal protein s6 kinase $\beta-1$ and eukaryotic translation initiation factor 4E-binding protein 1 (4E-BP1) compared with WT BMDMs (Fig. 5B). TIPE2 overexpression promoted $\mathrm{M} 2$ polarization and expression of M2 genes (Fig. 3D and E). Arginase I and arginase II expression was significantly increased in BMDMs with ectopic TIPE2 expression following IL-4 challenge (Fig. 5C), which could be responsible for the arginine-urea cycle dependence on mTORC1 inhibition (Fig. 5D) (21). Exogenous nitric oxide (NO) partly decreased IL-10 production under treatment with IL-4, whereas NO additionally promoted M1 inflammation induced by LPS and IFN $\gamma$. TIPE2 overexpression was able to reverse these phenotypes induced by exogenous NO (Fig. 5E). Conversely, in BMDMs with TSC1 deletion, constitutive 
A


sh-CTRL

Sh-TIPE2
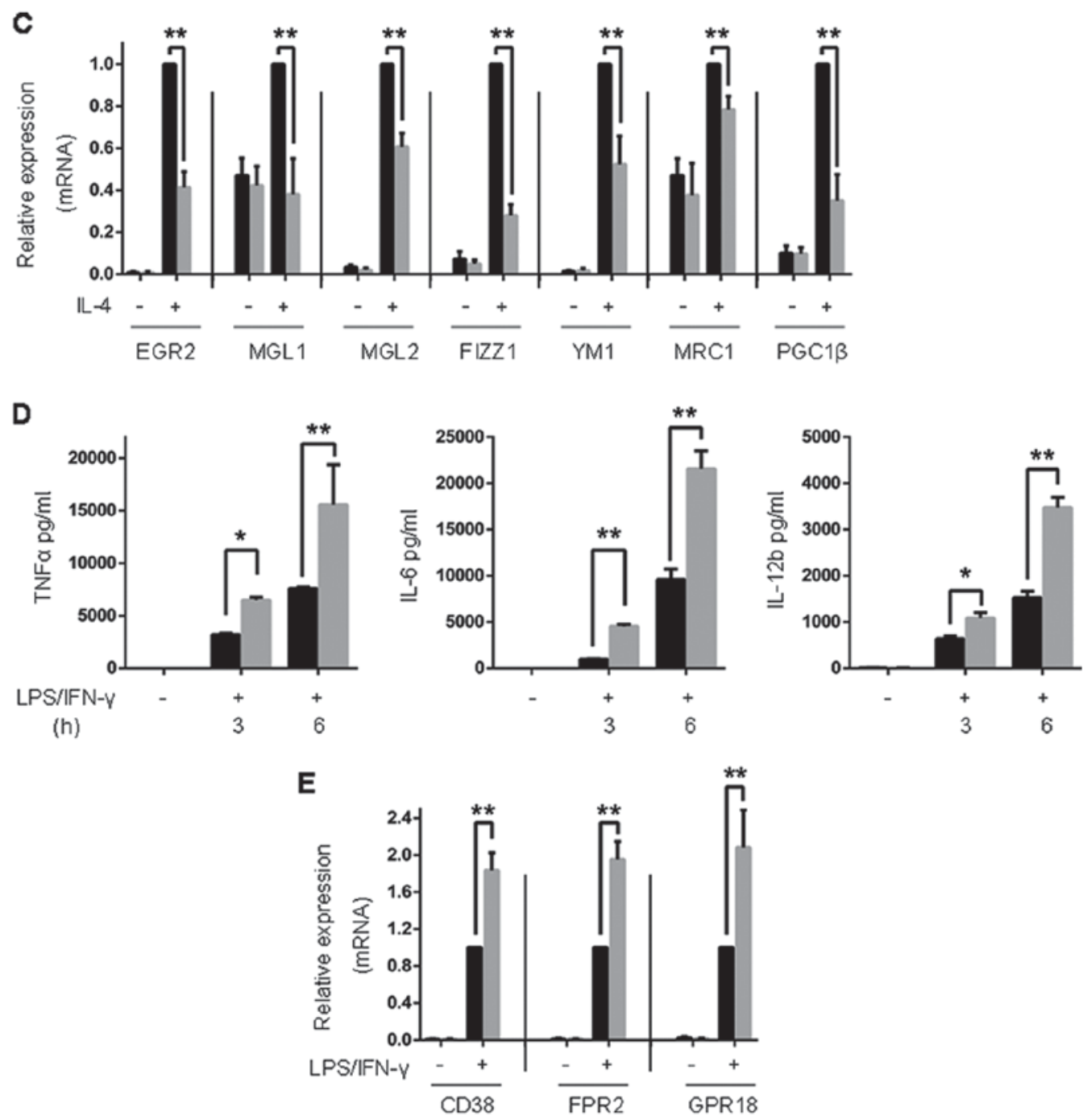

Figure 2. BMDMs with TIPE2 deletion have defective M2 polarization and enhanced responses to LPS/ IFN- $\gamma$ challenge. (A) BMDMs were transduced with TIPE2 shRNA. TIPE2 expression levels were determined by immuno blotting and reverse transcription-quantitative polymerase chain reaction analysis. (B) Measurement of IL-10 secretion in BMDMs by ELISA analysis following treatment with IL-4 $(20 \mathrm{ng} / \mathrm{ml})$ for 3 and $6 \mathrm{~h}$. (C) Expression of M2 genes in BMDMs following treatment with IL-4 (20 ng/ml) for 24h. (D) Measurement of TNF $\alpha$, IL-6 and IL-12b secretion in BMDMs by ELISA analysis following treatment with LPS $(10 \mathrm{ng} / \mathrm{ml})$ and IFN- $\gamma(100 \mathrm{U} / \mathrm{ml})$ for 3 and $6 \mathrm{~h}$. (E) Expression of M1 genes in BMDMs following treatment with LPS (10 ng/ml) and IFN $-\gamma(100 \mathrm{U} / \mathrm{ml})$ for $24 \mathrm{~h}$. Statistics were performed on pooled data from three independent experiments. Error bars represent the standard deviations of the means. ${ }^{*} \mathrm{P}<0.05,{ }^{* *} \mathrm{P}<0.01$. shRNA, short hairpin RNA; CTRL, control; TIPE2, tumor necrosis factor $\alpha$-induced protein 8-like protein 2; BMDMs, bone marrow-derived macrophages; LPS, lipopolysaccharide; IFN- $\gamma$, interferon- $\gamma$; EGR2, E3 SUMO protein ligase EGR2; MGL1, C-type lectin domain family 10 member A; MGL2, monoglyceride lipase; FIZZ1, resistin-like $\alpha$; YM1, chitinase-like protein 3; MRC1, macrophage mannose receptor 1; PGC1 $\beta$, peroxisome proliferator-activated receptor gamma coactivator $1-\beta ; \mathrm{CD} 38$, cluster of differentiation 38 ; FPR2, formyl peptide receptor 2 ; GPR18, N-arachidonyl glycine receptor; TNF $\alpha$, tumor necrosis factor- $\alpha$; IL, interleukin. 
A

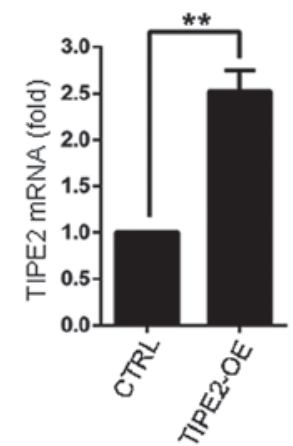

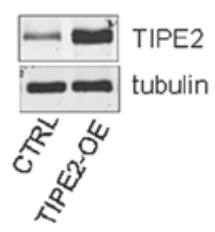

CTRL

C


B
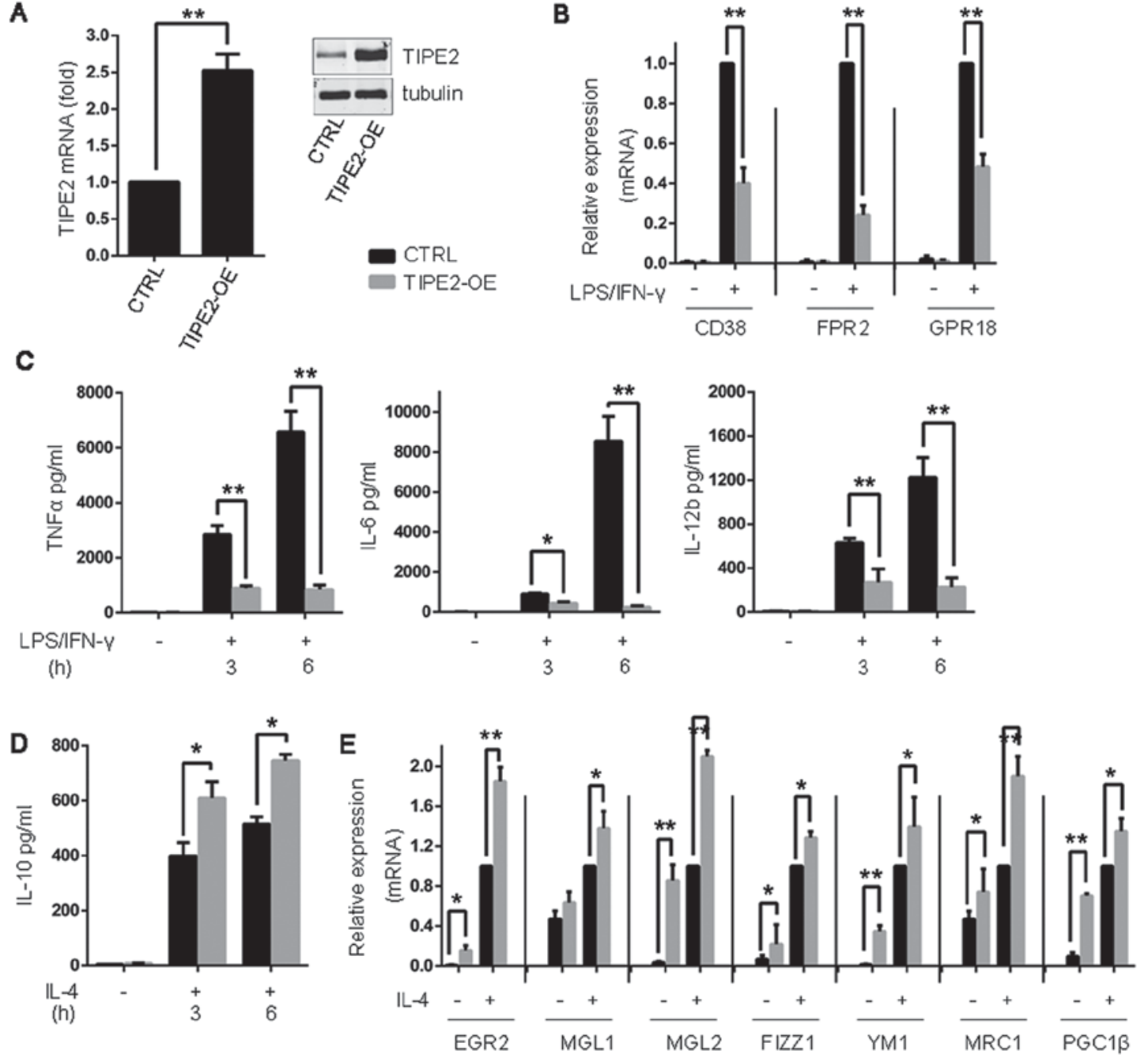

Figure 3. TIPE2 overexpression promotes M2 polarization and disturbs M1 inflammation. (A) BMDMs were transduced with exogenous TIPE2. TIPE2 expression levels were determined by immunoblotting and reverse transcription-quantitative polymerase chain reaction analysis. (B) Expression of M1 genes in BMDMs following treatment with LPS $(10 \mathrm{ng} / \mathrm{ml})$ and IFN- $\gamma(100 \mathrm{U} / \mathrm{ml})$ for $24 \mathrm{~h}$. (C) Measurement of TNF $\alpha$, IL-6 and IL-12b secretion in BMDMs by ELISA analysis following treatment with LPS $(10 \mathrm{ng} / \mathrm{ml})$ and IFN- $\gamma(100 \mathrm{U} / \mathrm{ml})$ for 3 and $6 \mathrm{~h}$. (D) Measurement of IL-10 secretion in BMDMs by ELISA following treatment with IL-4 (20 ng/ml) for $3 \mathrm{~h}$ and $6 \mathrm{~h}$. (E) Expression of M2 genes in BMDMs following treatment with IL-4 for $24 \mathrm{~h}$. Statistics were performed on pooled data from three independent experiments. Error bars represent the standard deviations of the means. ${ }^{*} \mathrm{P}<0.05,{ }^{* *} \mathrm{P}<0.01$. BMDMs, bone marrow-derived macrophages; TIPE2, tumor necrosis factor $\alpha$-induced protein 8-like protein 2; CTRL, control vector; TIPE2-OE, TIPE2 overexpression; LPS, lipopolysaccharide; CD38, cluster of differentiation 38; FPR2, formyl peptide receptor 2; GPR18, N-arachidonyl glycine receptor; IFN $\gamma$, interferon- $\gamma$; TNF $\alpha$, tumor necrosis factor- $\alpha$; IL, interleukin; EGR2, E3 SUMO protein ligase EGR2; MGL1, C-type lectin domain family 10 member A; MGL2, monoglyceride lipase; FIZZ1, resistin-like $\alpha$; YM1, chitinase-like protein 3; MRC1, macrophage mannose receptor 1; PGC1 $\beta$, peroxisome proliferator-activated receptor gamma coactivator $1-\beta$.

mTORC1 activity impaired IL-4-induced M2 polarization (Fig. 5C and E-G), and obstructed IRS-1/2 degradation, and AktT308 and S473 activation (Fig. 5H). BMDMs with TSC1 deletion through treatment with rapamycin and IL-4, or BMDMs with TSC1 deletion and TIPE2 overexpression, were able torescue M2 responses (Fig. 5C-E). The results of the present study demonstrated that TIPE2 overexpression weakened the activation of mTORC1 pathway, which accelerated IL-4-induced M2 polarization.

\section{Discussion}

In response to numerous microenvironmental signals, including different intracellular pathogens or direct interactions between inflammatory cells, macrophages primarily undergo M1 (classical) or M2 (alternative) activation, and exhibit different functional states of polarization (4). TIPE2 acts as a negative regulator of innate immunity $(14,15,22,23)$, althoughits role in macrophage polarization remains largely unknown. In the present study, an inverse expression pattern of TIPE2 in murine M1 and M2 macrophages was observed. BMDMs following LPS and IFN $\gamma$ stimulation exhibited markedly decreased TIPE2 expression, which was consistent with a previous report (23). However, IL-4-induced M0 macrophages polarized into M2 macrophages in line with elevated TIPE2 expression levels. The present study additionally identified the regulatory role of TIPE2 as an activator of M2 marker expression, and demonstrated that alterations in TIPE2 expression led to an interchange between macrophage subtypes.

TIPE2 deficiency facilitates M1 inflammation (14), whereas exogenous TIPE2 overexpression enhances M2 
A

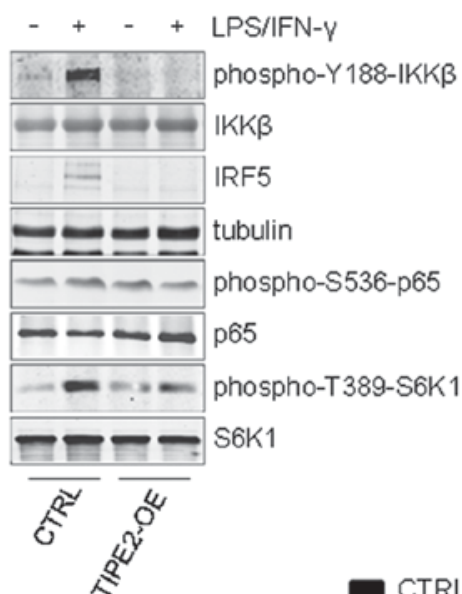

B

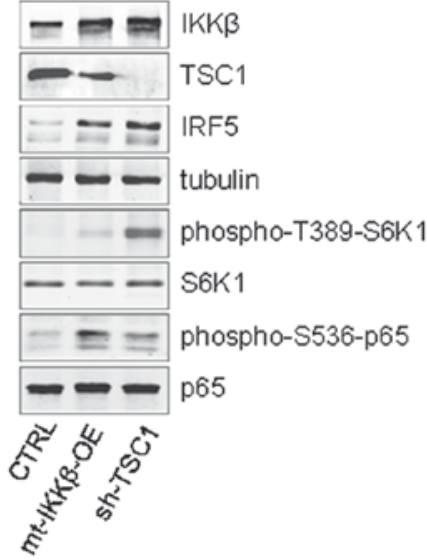

$m+-I K K \beta-O E$

口 $\mathrm{mt}-\mathrm{IKK} \beta-\mathrm{OE}+\mathrm{TIPE} 2-\mathrm{OE}$

$\otimes m t-I K K \beta-O E+T I P E 2-O E+s h-T S C 1$
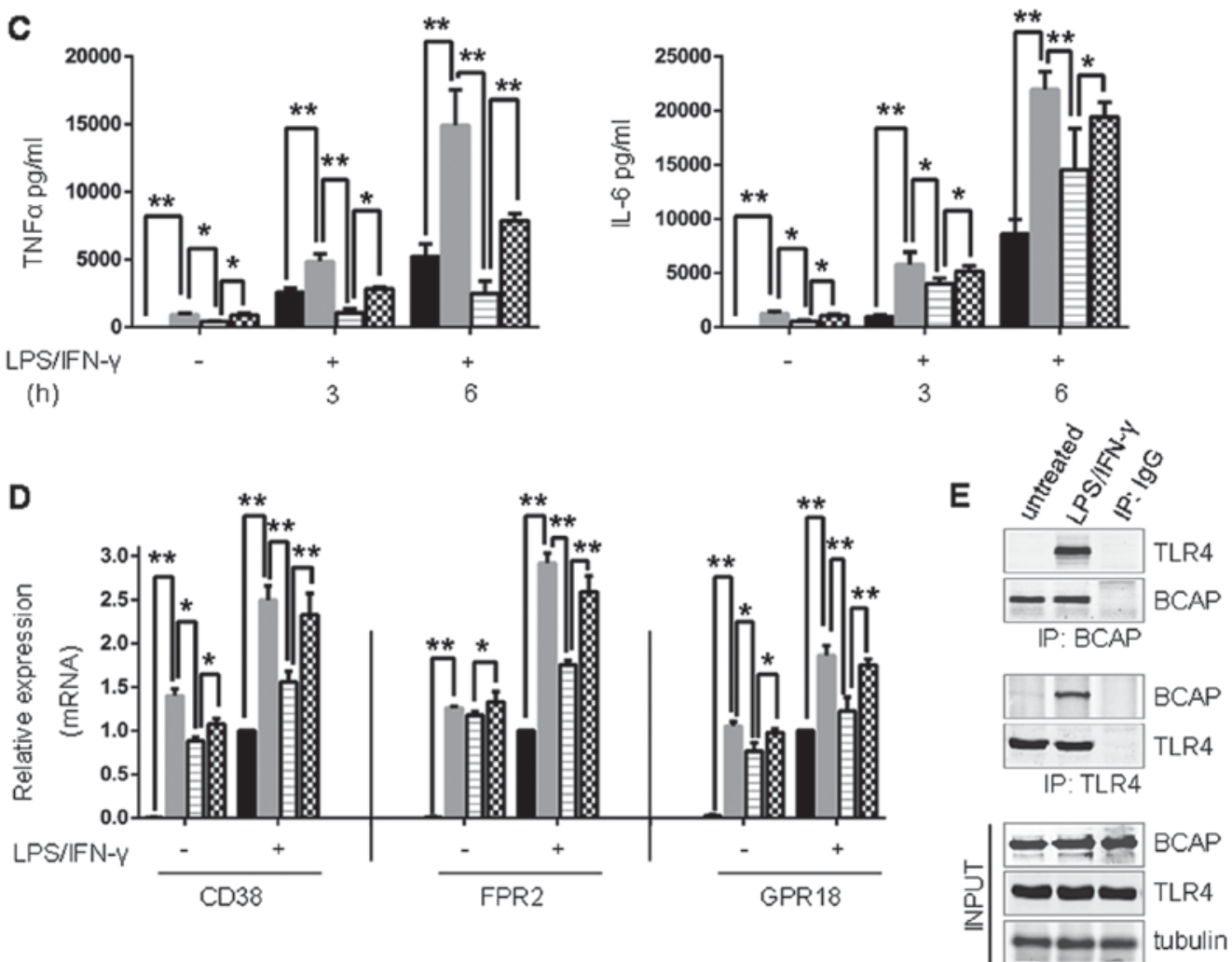

Figure 4. Ectopic TIPE2 attenuates M1 polarization via disturbance of the IKK $\beta$ and mTORC1 pathways. (A) The expression levels of IKK $\beta$, phospho-Y188-IKK $\beta$, IRF5, p65, phospho-S536-p65, S6K1, phospho-T389-S6K1 and tubulin were determined by immunoblotting. (B) The expression levels of IKK $\beta$, IRF5, p65, phospho-S536-p65, TSC1, S6K1, phospho-T389-S6K1 and tubulin were determined by immunoblotting. (C) Measurement of TNF $\alpha$ and IL-6 secretion by ELISA following treatment with LPS $(10 \mathrm{ng} / \mathrm{ml})$ and IFN- $\gamma(100 \mathrm{U} / \mathrm{ml})$ for 3 and $6 \mathrm{~h}$. (D) Expression of M1 genes following treatment with LPS (10 ng/ml) and IFN- $\gamma$ $(100 \mathrm{U} / \mathrm{ml})$ for $24 \mathrm{~h}$. (E) BCAP formed a complex with TLR4 and TLR4 formed a complex with BCAP in BMDMs, prior to and following LPS (10 ng/ml) and IFN- $\gamma(100 \mathrm{U} / \mathrm{ml})$ stimulation. Statistics were performed on pooled data from three independent experiments. Error bars represent the standard deviations of the means. ${ }^{*} \mathrm{P}<0.05,{ }^{* *} \mathrm{P}<0.01$. phospho, phosphorylated; CTRL, control vector; TIPE2, tumor necrosis factor $\alpha$-induced protein 8 -like protein 2 ; OE, overexpression; sh, short hairpin RNA; IKK $\beta$, inhibitor of nuclear factor- $\mathrm{kB}$ subunit $\beta$; mt-IKK $\beta$, IKK $\beta$ with K171T mutation; IRF5, interferon regulatory factor 5; p65, transcription factor p65; mTORC, serine/threonine-protein kinase mTOR complex 1; S6K1, ribosomal protein s6 kinase $\beta$-1; TSC1, hamartin; IL-6, interleukin-6; TNF $\alpha$, tumor necrosis factor- $\alpha$; IFN $\gamma$, interferon- $\gamma$; LPS, lipopolysaccharide; BCAP, B-cell receptor-associated protein; TLR4, Toll-like receptor 4; BMDMs, bone marrow-derived macrophages; CD38, cluster of differentiation 38; FPR2, formyl peptide receptor 2; GPR18, N-arachidonyl glycine receptor.

polarization. The TLR-TAK1-IKK $\beta$ pathway promotes the transcription of M1-polarizing genes via IRF5 and NF- $\mathrm{B}$ activation (18-20). TIPE2 disrupted TAK1-TGF- $\beta$-activated kinase 1 and MAP3K7-binding protein (TAB)1-TAB2 formation, and subsequently inhibited the activation of TAK1 and its downstream molecules (16). In the present study, BMDMs with
mt-IKK $\beta$ (K171T), which represent constitutive activation of IKK $\beta$, exhibited increased M1-like functional activation, even prior to LPS/IFN $\gamma$ challenge. Exogenous TIPE2 and mt-IKK $\beta$ expression in M0 macrophages illustrated that ectopic TIPE2 partially reduced glycolytic flux and reversed the course of M1 polarization stimulated by LPS/IFN $\gamma$. 
A
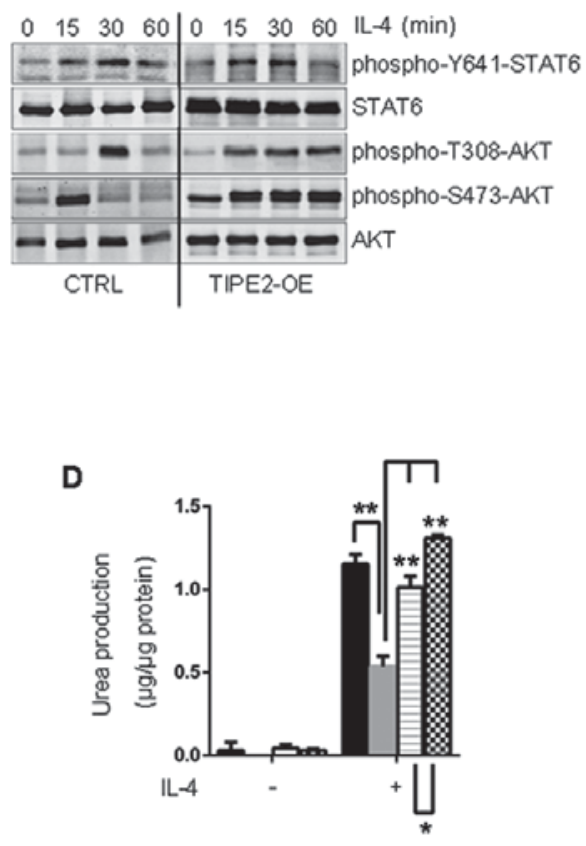

B

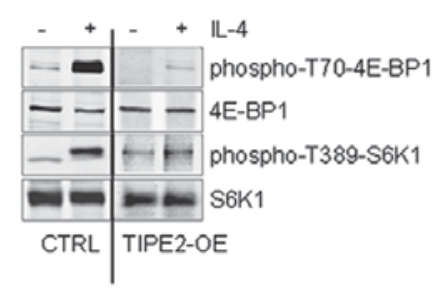

C

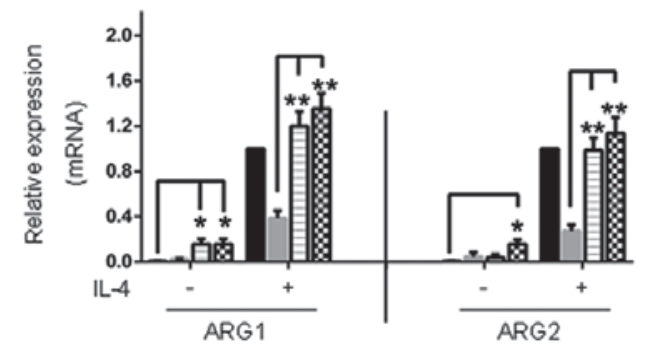

- CTRL TIPE2-OE
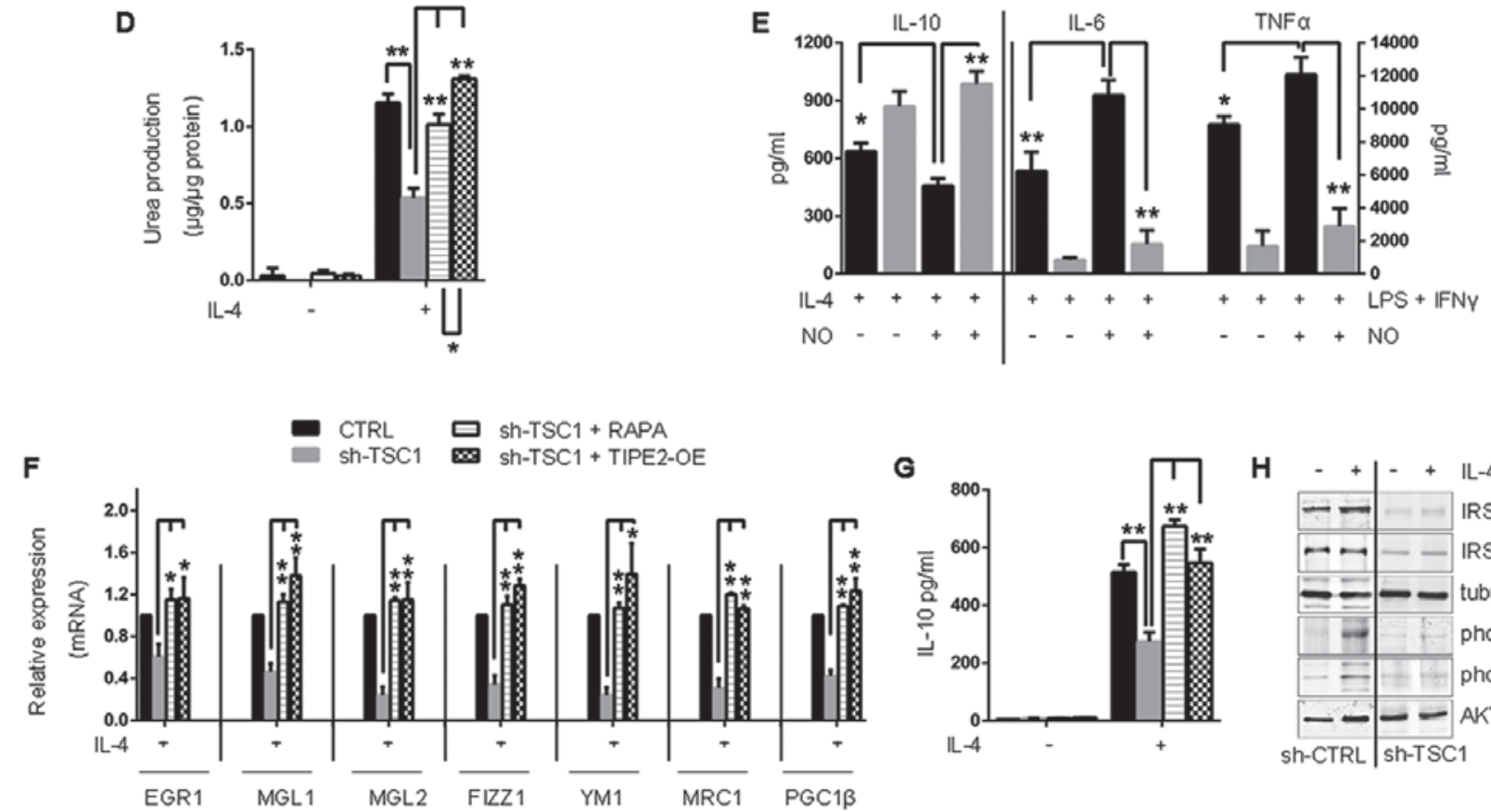

G

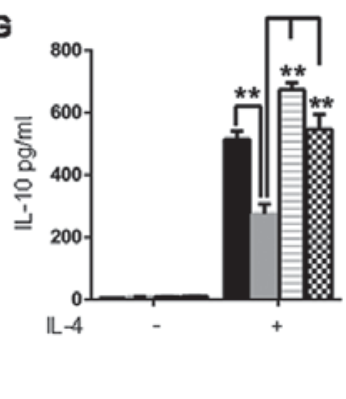

H

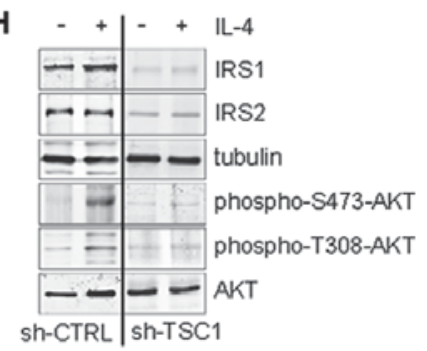

Figure 5. TIPE2 overexpression promotes M2 polarization induced by IL-4. (A) The expression levels of AKT, phospho-T308-AKT, phospho-S473-AKT, STAT6 and phospho-Y641-STAT6 were determined by immunoblotting. (B) The expression levels of 4E-BP1, phospho-T70-4E-BP1, S6K1 and phospho-T389-S6K1 were determined by immunoblotting. (C) The expression levels of the ARG I and II genes following treatment with IL-4 (20 ng/ml) for $24 \mathrm{~h}$ were determined by RT-qPCR analysis. (D) Measurement of urea production normalized to the total protein in BMDMs, Following treatment with IL-4 (20 ng/ml) for $6 \mathrm{~h}$. (E) Measurement of IL-10, IL-6 and TNF $\alpha$ secretion by ELISA following treatment with IL-4 (20 ng/ml), NO (100 $\mu \mathrm{M})$ or LPS (10 ng/ml) and IFN- $\gamma(100 \mathrm{U} / \mathrm{ml})$ for $6 \mathrm{~h}$. (F) The expression levels of M2 genes following treatment with IL-4 (20 ng/ml) for $24 \mathrm{~h}$ were determined by RT-qPCR. (G) Measurement of IL-10 secretion by ELISA following treatment with IL-4 $(20 \mathrm{ng} / \mathrm{ml})$ for $6 \mathrm{~h}$. (H) The expression levels of IRS1, IRS2, AKT, phospho-T308-AKT, phospho-S473-AKT and tubulin were determined by immunoblotting. Statistics were performed on pooled data from three independent experiments. Error bars represent the standard deviations of the means. ${ }^{*} \mathrm{P}<0.05,{ }^{* *} \mathrm{P}<0.01$. phospho, phosphorylated; CTRL, control vector; OE, overexpression; sh, short hairpin RNA; RAPA, rapamycin; STAT6, signal transducer and activator of transcription 6; AKT, RAC- $\alpha$ serine/threonine-protein kinase; TIPE2, tumor necrosis factor $\alpha$-induced protein 8-like protein 2; IL, interleukin; 4E-BP1, eukaryotic translation initiation factor 4E-binding protein 1; S6K1, ribosomal protein S6 kinase $\beta$-1; ARG, arginase; RT-qPCR, reverse transcription-quantitative polymerase chain reaction; LPS, lipopolysaccharide; IFN $\gamma$, interferon- $\gamma$; NO, nitric oxide; TNF $\alpha$, tumor necrosis factor- $\alpha$; EGR2, E3 SUMO protein ligase EGR2; MGL1, C-type lectin domain family 10 member A; MGL2, monoglyceride lipase; FIZZ1, resistin-like $\alpha$; YM1, chitinase-like protein 3; MRC1, macrophage mannose receptor 1; PGC1 $\beta$, peroxisome proliferator-activated receptor gamma coactivator $1-\beta$; IRS, insulin receptor substrate; TSC1, hamartin.

Except for the TAK1-IKK $\beta$ pathway, TIPE2 disturbed the activation of TLR4-BCAP-mTORC1 signaling and inhibited the transcription of M1 genes. Therefore, mTORC1 and IKK $\beta$ may be able to coordinate M1 polarization following stimulation with LPS/IFN $\gamma$.

The role of TIPE2 in inhibiting the activation of M1 inflammation identified in the present study is important, due toits well-documented suppression of TAK1-IKK $\beta$ (16) and TLR4 activation (22). In the present study, the expression of TIPE2 was elevated in IL-4-induced M2 polarization. Exogenous TIPE2 expression accelerated the process of M2 polarization by IL-4. Overexpression of TIPE2 elevated the arginase I and arginase II levels, which may accelerate the conversion of L-arginine into urea $(24,25)$. Exogenous NO partially inhibited the production of IL-10 under treatment with IL-4, and was able to promote M1 inflammation induced by LPS and IFN $\gamma$, which may be caused by the activation of mTORC1 (21). TIPE2 was able to reverse these processes induced by NO. These observations were consistent with the hypothesis that arginases and NO synthases may compete for L-arginine, the common substrate of urea, or NO synthesis, which determines the fate of macrophage polarization $(24,26-28)$. In addition, arginine metabolism was demonstrated to servean important role in manipulating the distinct M1/M2 phenotypes $(26,27,29)$. 
In conclusion, TIPE2 serves an important role in orchestrating the differential polarization status of macrophages, via negative regulation of mTORC 1 activation and accelerated conversion of arginine to urea. The influence of TIPE2 on the elevation of arginase expression and the effects of $\mathrm{NO}$ or urea on M1/M2 polarization remain unclear. Further investigation of how signal transduction downstream of TIPE2 affects arginine metabolism may reveal the mechanism through which TIPE2 enhances IL-4 induced M2 polarization.

\section{Acknowledgements}

The present study was supported by grants from the Projects of Medical and Health Technology Development Program in Zhejiang Province, China (grant nos. 2016KYB135 and 2016KYB171).

\section{References}

1. Mosser DM and Edwards JP: Exploring the full spectrum of macrophage activation. Nat Rev Immunol 8: 958-969, 2008.

2. Ginhoux F and Jung S: Monocytes and macrophages: Developmental pathways and tissue homeostasis. Nat Rev Immunol 14: 392-404, 2014.

3. Varol C, Mildner A and Jung S: Macrophages: Development and tissue specialization. Annu Rev Immunol 33: 643-675, 2015.

4. Italiani P and Boraschi D: From Monocytes to M1/M2 Macrophages: Phenotypical vs. functional differentiation. Front Immunol 5: 514, 2014

5. Saeed S, Quintin J, Kerstens HH, Rao NA, Aghajanirefah A, Matarese F, Cheng SC, Ratter J,Berentsen K, van der Ent MA, et al: Epigenetic programming of monocyte-to-macrophage differentiation and trained innate immunity. Science 345: 1251086, 2014

6. Byles V, Covarrubias AJ, Ben-Sahra I,Lamming DW, Sabatini DM, Manning BD and Horng T: The TSC-mTOR pathway regulates macrophage polarization. Nat Commun 4: 2834, 2013.

7. Covarrubias AJ, Aksoylar HI and Horng T: Control of macrophage metabolism and activation by mTOR and Akt signaling. Semin Immunol 27: 286-296, 2015.

8. Weichhart T, Hengstschläger M and Linke M: Regulation of innate immune cell function by mTOR. Nat Rev Immunol 15: 599-614, 2015.

9. Huang SC, Smith AM, Everts B, Colonna M, Pearce EL Schilling JD and Pearce EJ: Metabolic reprogramming mediated by the mTORC2-IRF4 signaling axis is essential for macrophage alternative activation. Immunity 45: 817-830, 2016.

10. Brown J, Wang H, Suttles J, Graves DT and Martin M: Mammalian target of rapamycin complex 2 (mTORC2) negatively regulates Toll-like receptor 4-mediated inflammatory response via FoxO1. J Biol Chem 286: 44295-44305, 2011.

11. Covarrubias AJ, Aksoylar HI, Yu J, Snyder NW, Worth AJ, Iyer SS, Wang J, Ben-Sahra I, Byles V, Polynne-Stapornkul T, et al: Akt-mTORC1 signaling regulates Acly to integrate metabolic input to control of macrophage activation. Elife 5: pii: e11612, 2016.

12. Sun H, Gong S, Carmody RJ, Hilliard A, Li L, Sun J, Kong L, $\mathrm{Xu} \mathrm{L}$, Hilliard B, Hu S, et al: TIPE2, a negative regulator of innate and adaptive immunity that maintains immune homeostasis. Cell 133: 415-426, 2008

13. Zhu Y, Tao M, Wu J, Meng Y, Xu C, Tian Y, Zhou X, Xiang J, Zhang $\mathrm{H}$ and Xie Y: Adenovirus-directed expression of TIPE2 suppresses gastric cancer growth via induction of apoptosis and inhibition of AKT and ERK1/2 signaling. Cancer Gene Ther 23: 98-106, 2016.
14. Lou Y, Zhang G, Geng M, Zhang W, Cui J and Liu S: TIPE2 negatively regulates inflammation by switching arginine metabolism from nitric oxide synthase to arginase. PLoS One 9: e96508, 2014.

15. Li F, Zhu X, Yang Y, Huang L and Xu J: TIPE2 alleviates systemic lupus erythematosus through regulating macrophage polarization. Cell Physiol Biochem 38: 330-339, 2016.

16. Oho M, Nakano R, Nakayama R, Sakurai W, Miyamoto A, Masuhiro Y and Hanazawa S: TIPE2 (tumor necrosis factor alpha-induced protein 8-like 2) is a novel negative regulator of TAK1 signal. J Biol Chem 291: 22650-22660, 2016.

17. Livak KJ and Schmittgen TD: Analysis of relative gene expression data using real-time quantitative PCR and the 2(-Delta Delta C(T)) method. Methods 25: 402-408, 2001.

18. Ren J, Chen X and Chen ZJ: IKK $\beta$ is an IRF5 kinase that instigates inflammation. Proc Natl Acad Sci USA 111: 17438-17443, 2014.

19. Lopez-Pelaez M, Lamont DJ, Peggie M, Shpiro N, Gray NS and Cohen P: Protein kinase IKK $\beta$-catalyzed phosphorylation of IRF5 at Ser462 induces its dimerization and nuclear translocation in myeloid cells. Proc Natl Acad Sci USA 111: 17432-17437, 2014.

20. Mihaly SR, Morioka S, Ninomiya-Tsuji J and Takaesu G: Activated macrophage survival is coordinated by TAK1 binding proteins. PLoS One 9: e94982, 2014.

21. Chantranupong L, Scaria SM, Saxton RA, Gygi MP, Shen K, Wyant GA, Wang T, Harper JW, Gygi SP and Sabatini DM: The CASTOR proteins are arginine sensors for the MTORC1 pathway. Cell 165: 153-164, 2016.

22. Sun H, Zhuang G, Chai L, Wang Z, Johnson D, Ma Y and Chen YH: TIPE2 controls innate immunity to RNA by targeting the phosphatidylinositol 3-kinase-Rac pathway. J Immunol 189: 2768-2773, 2012

23. Wang Z, Fayngerts S, Wang P, Sun H, Johnson DS, Ruan Q, Guo $\mathrm{W}$ and Chen YH: TIPE2 protein serves as a negative regulator of phagocytosis and oxidative burst during infection. Proc Natl Acad Sci USA 109: 15413-15418, 2012.

24. Munder M: Arginase: An emerging key player in the mammalian immune system. Br J Pharmacol 158: 638-651, 2009.

25. Nissim I, Luhovyy B, Horyn O, Daikhin Y, Nissim I and Yudkoff M: The role of mitochondrially bound arginase in the regulation of urea synthesis: Studies with [U-15N4]arginine, isolated mitochondria, and perfused rat liver. J Biol Chem 280: 17715-17724, 2005.

26. Comalada M, Yeramian A, Modolell M,Lloberas J and Celada A: Arginine and macrophage activation. Methods Mol Biol 844: 223-235, 2012.

27. Rath M, Müller I, Kropf P, Closs EI and Munder M: Metabolism via arginase or nitric oxide synthase: Two competing arginine pathways in macrophages. Front Immunol 5: 532,2014

28. McNeill E, Crabtree MJ, Sahgal N, Patel J, Chuaiphichai S, Iqbal AJ, Hale AB, Greaves DR and Channon KM: Regulation of iNOS function and cellular redox state by macrophage Gch1 reveals specific requirements for tetrahydrobiopterin in NRF2 activation. Free Radic Biol Med 79: 206-216, 2015.

29. Thomas AC and Mattila JT: 'Of mice and men': Arginine metabolism in macrophages. Front Immunol 5: 479, 2014. 